
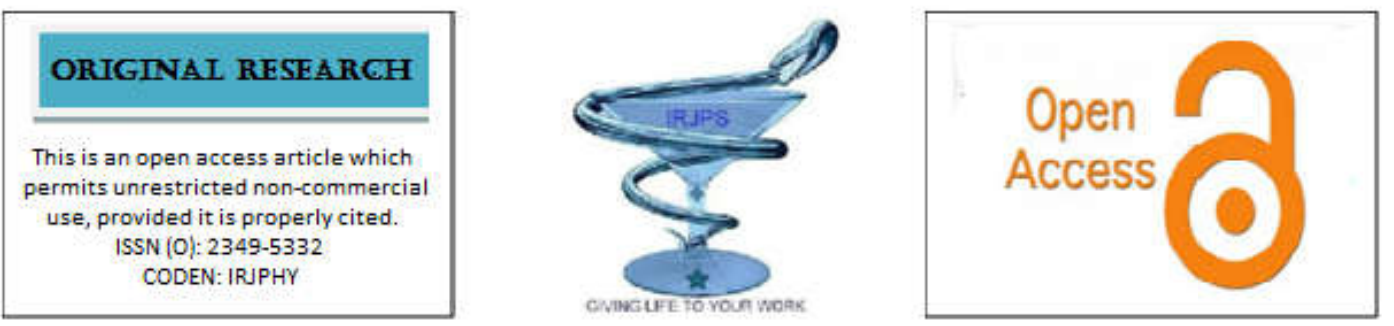

\title{
ANTIBIOTIC SUSCEPTIBILITY OF METHICILLIN RESISTANT STAPHYLOCOCCUS AUREUS IN ORTHOPAEDIC PATIENTS IN RURAL SETTINGS
}

\author{
Ulhas Dudhekar, Abhiram Awasthi, Shivshankar Jadhav*, Mukund Dhaniwala, Ankur Salwan, Vivek \\ Jadawala
}

Department of Orthopaedics, Jawaharlal Nehru Medical College, Datta Meghe Institute of Medical Sciences, Wardha, Maharashtra, India

Submitted on: 29.12.2020;

Revised on: 24.01.2021;

Accepted on: 30.01 .2021

\begin{abstract}
:
Introduction: Throughout the world, Methicillin Resistant Staphylococcus aureus (MRSA) infection is a welldocumented public health issue.Due to extensive use of antibiotics including beta lactams and others, resistance to antimicrobials has grown over time. Due to the genetic modifications in micro-organisms including MRSA, multiple new strains of resistant bacteria have emerged.

Materials and Methods: 50 patients with infected wounds who came to the outpatient department of AVBRH hospital Sawangi, Wardha. Pus culture sensitivity swabs of all the patients were sent and based on the reports of antibiotic susceptibility patients were classified. Specimen were taken mostly from surgical wounds, abscess pus and trauma patients.

Results: Out of 50 patients $32(64 \%)$ patients were found to harbour S.aureus. and out of those 32 patients 23 had MRSA. Patients of age 10-30 years (12 patients) were found to have more chances of infection with MRSA as compared to 30-60 age group (10 patients) and older age group ( 1 patient). The results also showed that out of 50 patients, 29 were sensitive to vancomycin, 31 patients were sensitive to amikacin, 17 to clindamycin, 16 to ciprofloxacin.

Conclusion:The study concluded that majority of patients with infected wounds harbour MRSA and most of the patients with MRSA infection were susceptible to vancomycin (21 patients $91.30 \%$ ). Therefore it is advisable to use susceptible antibiotics in infection control practices.
\end{abstract}

KEYWORDS: Antibiotic susceptibility, MRSA, Staphylococcus Aureus

Corresponding author: S. Jadhav

Mob no: 8669018503

Email id: shivjadhav727@gmail.com
Indian Research Journal of Pharmacy and Science; 26(2020)2466-2471; Journal Home Page: https://www.irips.in DOI: 10.21276/irjps.2020.7.4.8 


\section{INTRODUCTION}

Multi drug resistance in staphylococcus strains was initially described in 1960s. In the last decade these strains have grown rapidly as a cause of hospital acquired infections and have caused potentially life threatening conditions such as pneumonias, endocarditis, septic arthritis and toxic shock syndromes ${ }^{1}$. Many identifiable risk factures have been reported in the literature regarding the infection of MRSA such as immune-suppression, diabetes mellitus, young age, long term use of steroids, non-healing ulcers ${ }^{2}$.

Initially MRSA was described in hospital acquired infections hence it was named as Health care associated MRSA (HA-MRSA). There have been 2 genetically distinct varietiesof MRSA described namely the community associated MRSA and livestock associated $\mathrm{MRSA}^{3}$. Also the evolving resistance even to the latest group of antibiotics namely the linezolid group has worsened the crisis associated with MRSA ${ }^{4,5}$.

As compared to the methicillin sensitive MRSA, the hospital acquired variant of MRSA is associated with increased morbidity and mortality of the infected patient and has more socioeconomic burden over him6. The EAARS (European antimicrobial resistance surveillance system) has reported the prevalence of hospital acquired MRSA between $1 \%$ and $24 \%$ in acute and long term hospital stay. ${ }^{7}$. In Indian scenario, the prevalence of MRSA has increased from 12 percent in 1992 to 80.8 percent in $1999^{8}$.

In general practice the 2 distinct variety of MRSA namely the CA-MRSA and the HA-MRSA differ each other in susceptibility to antibiotics. The CAMRSA is generally resistant to Beta lactam antibiotics and susceptible to fluroquinolones, cotrimoxazole, and chloramphenicol whereas the HA-MRSA is resistant to fluroquinolones and clindamycin and is usually sensitive to vancomycin ${ }^{9}$. But a distinct differentiation between HA-MRSA and CA-MRSA has not been possible yet. One differentiation has been found out regarding the PVL (Panton-Valentine Leukocidin) gene which generally encodes for pores forming cytotoxin, is commonly found in CA-MRSA and rarely found in HA-MRSA.

Methicillin resistance in staph is created by PBP2a protein which is encoded by MEC A gene which provides resistance to MRSA from most of the BLactam antibiotics ${ }^{10}$. In order to fight against MRSA and its drug resistance, further investigations and surveillance is needed. In this study the antibiotic susceptibility of MRSA to antibiotics in rural orthopaedic patients with control measures for infection have been discussed.

\section{MATERIAL AND METHODS:}

Study Design: This study was conducted at Acharya VinobhaBhave Rural Hospital (AVBRH), Sawangi, Wardha. Specimens collected from the patients with soft tissue and skin infection purulent wound discharges were then sent to the microbiology laboratory for further processing. Information in addition to the specimens like age, onset of discharge, sex, any history of antibiotic use and any other comorbidities like diabetes and/or hypertension or any other medical condition was taken. The study was conducted in the year Janauary2019-July 2020.

\section{Inclusion Criteria:}

Patient included who had -

i) active discharge and/or pus from the surgical site infection,

ii) abscess in the skin

\section{Exclusion Criteria:}

i) patients not giving informed consent,

ii) patients with gangrenous changes in wounds and

iii) mangled extremities.

\section{Sample:}

50 samples were taken from the abscess, infected surgical site wound which were collected using sterile swab sticks and sent in sterile culture bottles. Levine's technique was used for the preparation of the sites from where the samples was to be collected $^{11}$. This technique included rotating of the wound swab over 1-2 cm of the wound. Purulent discharges, pus collected from the surgical site wound and abscess were inoculated and cultured in the nutrient agar (NA) and mannitol salt agar (MSA) respectively and incubated for one day at 37 degree Celsius in aerobic environment. 
Golden yellow color bacterial colonies on MSA were visible typical of S.aureus, which were then gram stained and subjected to catalase test \& DNase test. Gram positive bacteria, fermenting mannitol appearing as grape like cluster that have catalase positive activity were then cultured in DNase agar and incubated for one day at 37 degree Celsius. HCL (1N) was introduced in the DNase agar plates. It was seen that the DNA was hydrolyzed which is the ability of S.aureus. Nutrient agar components include $0.5 \%$ of peptone extract, $0.3 \%$ beef extract, $1.5 \%$ agar, $0.5 \% \mathrm{Nacl}$, distilled water and $\mathrm{pH}$ is adjusted to neutal (7.4) at 25 degreeCelcius.

Antimicrobial testing for sensitivity was also performed. As per the Clinical and Laboratory Standards Institute (CLSI) guidelines, a KirbyBauer disk diffusion method using the Muller Hinton Agar (MHA) plate technique was used ${ }^{12}$. Antimicrobial disks with multiple drug classes were used for oxacillin, vancomycin and gentamycin. These were incubated for one day at 37 degree Celsius in MHA along with 2 percent $\mathrm{NaCl}$. For each antimicrobial testing an inhibition zone diameter was measured and labelled as resistant (R) and sensitive (S) after comparison

with mecA negative \&mecA positive S.aureus. These zones were interpreted as per CLSI guidelines. Hospital acquired infections were defined as infections occurring for more than three calendar days after admissions in the hospital.

Ethical clearance was obtained and every participant was explained about the study and informed consent was taken. For underage patients parents and/or legal guardians were asked to sign consent forms. Patients confidentiality along with the reports was maintained until the study was completed.

\section{RESULTS:}

Out of 50 specimen collected,38(76\%) are males and $12(24 \%)$ are females. Range of age group included in the study was 0 years to 90 years. Age groups between 30 year to 60 year were more in number. Of the 50 samples collected $30(60 \%)$ were S.aureus sensitive. 15(65.21\%) of males were MRSA sensitive and $7(100 \%)$ were MSSA sensitive (Table-1).

Table.1: Association of organisms with respect to age group and gender

\begin{tabular}{|l|c|c|c|c|c|}
\hline \multicolumn{2}{|c|}{ Variables } & MRSA (number and \%) & MSSA (number and \%) & $\begin{array}{l}\text { Others } \\
\text { (number and \%) }\end{array}$ & Total \\
\hline \multirow{3}{*}{ Sex } & Males & $15(65.21 \%)$ & $7(100 \%)$ & $16(80 \%)$ & $38(76 \%)$ \\
\cline { 2 - 6 } & Females & $8(34.79 \%)$ & $0(0 \%)$ & $4(20 \%)$ & $12(24 \%)$ \\
\cline { 2 - 6 } & Total & $23(100 \%)$ & $7(100 \%)$ & $20(100 \%)$ & $50(100 \%)$ \\
\hline \multirow{2}{*}{$\begin{array}{l}\text { Age } \\
\text { group }\end{array}$} & $0-30$ & $12(52.17)$ & $2(28.57)$ & $6(30 \%)$ & $20(40 \%)$ \\
\cline { 2 - 6 } & $30-60$ & $10(43.47 \%)$ & $4(57.14 \%)$ & $12(60 \%)$ & $26(52 \%)$ \\
\hline & $60-90$ & $1(4.36 \%)$ & $1(14.29 \%)$ & $2(10 \%)$ & $4(8 \%)$ \\
\hline
\end{tabular}

In association with antibiotic sensitivity, 21 patients $(91.30 \%) \& 7(100 \%)$ of MRSA \& MSSA are sensitive too Vancomycin, $19(82.60 \%)$ \& 5 (71.42\%) of MRSA \& MSSA were sensitive to Amikacin, $18(78.23 \%) \& 6(85.71 \%)$ of MRSA \& MSSA are sensitive to Clindamycin, $14(60.8 \%)$ \&6 $(85.71 \%)$ of MRSA \& MSSA are sensitive to Linezolid and $6(26.08 \%)$ \& 3 (42.45\%) of MRSA $\&$ MSSA are sensitive to Ciprofloxacin (Table-2).

The antibiotic sensitivity test for MRSA resulted 2
$(8.70 \%)$ resistant to Vancomycin, 4 (17.4\%) resistant to Amikacin, $5(21.7 \%)$ resistant to Clindamycin, 9 (39.2\%) are resistant too Linezolid, $17(73.92 \%)$ are resistant to Ciprofloxacin (Table2).

The antibiotic sensitivity test for MSSA resulted 2 (28.58\%) resistant to Amikacin, 1 (14.29\%) resistant to Clindamycin, 1 (14.29\%) are resistant too Linezolid, $4(57.5 \%)$ are resistant to Ciprofloxacin(Table-2). 
Table 2: Antimicrobial sensitivity of MRSA and MSSA to different antibiotics

\begin{tabular}{|l|c|c|c|c|c|}
\hline & Vancomycin & Amikacin & Clindamycin & Linezolid & Ciprofloxacin \\
\hline MRSA & $21 / 23$ & $19 / 23$ & $18 / 23$ & $14 / 23$ & $6 / 23$ \\
susceptibility & $(91.30)$ & $(82.60 \%)$ & $(78.23 \%)$ & $(60.8 \%)$ & $(26.08 \%)$ \\
\hline MSSA & $7 / 7$ & $5 / 7$ & $6 / 7$ & $6 / 7$ & $3 / 7$ \\
susceptibility & $(100 \%)$ & $(71.42 \%)$ & $(85.71 \%)$ & $(85.71 \%)$ & $(42.45)$ \\
\hline Others & $10 / 20$ & $9 / 20$ & $8 / 20$ & $11 / 20$ & $14 / 20$ \\
susceptibility & $(50 \%)$ & $(45 \%)$ & $(40 \%)$ & $(55 \%)$ & $(70 \%)$ \\
\hline
\end{tabular}

\section{DISCUSSION:}

Staphylococcal cassette chromosome mec (SCCmec) contains an exogenous gene (mecA) which has been traced from the evolution of methicillin resistant $\mathrm{S}$. aureus which is under control of a repressor and a transducer namely MecI and MecRI which are the signalling proteins or regulatory proteins in the blaZ system ${ }^{13}$. Penicillin-binding protein (PBP2a) which is a peptidoglycan transpeptidase and is coded by mecA gene which confers resistance to all $\beta$-lactam antibiotics like penicillins, cephalosporins \& carbapenems. A variant of SCCmec type II \& III have higher resistance potential due presence of more resistance genes. The acronym MRSA - or Oxacillin- resistant S.aureus (ORSA) is because of the presence of PBP2a or mecA positivity which can be typed using the methicillin or oxacillin ${ }^{14}$.

$\mathrm{S}$. aureus isolation rate from the 50 samples was $30 / 50$ samples according to our data. Sex of the patients was not associated with the presence of S. aureus. Nature of patient sampled included surgical wound and diabetic patients. Due to long hospital stay these patients are at increased risk of developing bacterial diseases invasively and this results in colonization of MRSA.

The isolation rate from wound and abscess discharges was the highest $(100 \%)$ and from the pus and blood samples was about 62.1 percent and 40 percent, respectively(15). Eyob YohanessGaroy et al conducted a study in which they isolated 82 S.aureus samples out of which 59 (72 percent) were MRSA, 16 (19.5 percent) were MSSA, while the remaining 7 ( 8.5 percent) were MISA ${ }^{15}$.

Prevalence of MRSA has been associated to many factors including different study design, specimen types, procedures in the laboratory, population and the duration of study. For example, some of the studies depend upon the phenotypic MRSA detection procedures while the other procedures depend upon DNA (MLST- multilocus sequence typing), sequencing of genome, micro arrays , convention technique - PCR) which being gold standard for mecA. PCR technique which relies on detection of the genotype reports lower MRSA prevalence $^{16}$. There are other studies which have demonstrated that cefoxitin disk diffusion test have better performance $\&$ the oxacillin assays have acceptable specificity, sensitivity, negative and positive predictive value (NPV-PPV). In major southern districts of Tamil Nadu, 250 strains of methicillin resistant S.aureus were isolated from the 90 strains. Clinical and carrier subjects of all strains are sensitive to vancomycin ${ }^{14}$. CA-MRSA isolates are increasing in India recently. D' Souza et al in their study studied 412 confirmed cases of MRSA and out of which $54 \%$ were true CAMRSA having the SCCmec and SCCmec genes ${ }^{17}$. Arora et al in their study in north India found that the prevalence of MRSA was $46 \%$ and MRSA isolates are more resistant to other antibiotics than $\mathrm{MSSA}^{18}$.

\section{LIMITATION:}

The data presented in this study is from where information from AMR is limited. The true case load of hospitals is still unknown. The data collected and interpreted results should not be applied to the entire country. The difference between HA-MRSA \& CA-MRSA is not clear. Thus, the source of infection is not certain. Also some parameters like place of residency, the days of hospital stay of the patient, any history of antibiotics intake and any history of antimicrobial usage. Even after so many limitations, this study provides important information regarding MRSA 
and patterns of AMR associated with it. Thus, this data implies an important quality care for the patients, antibiotic selection, good practice of infection control.

\section{CONCLUSION:}

To summarize, there is prevalence of MRSA in the hospital with increase MRSA positivity in strains of S.aureus to antibiotics. Although the drug of choice for MRSA infection still is Vancomycin. Their should be monitoring of the patterns of MRSA susceptible to the antibiotics, helping in formulation for antibiotic policy which might be helpful in near future for decreasing the prevalence of MRSA infection. Their should be a tracking system to gather the data on emerging trend of infection which can be used later for designing infection control practices and the use of antimicrobial agents.

ACKNOWLEDGEMENT: We would like to acknowledge the personnel at Department of Orthopaedics, Datta Meghe Institute of Medical Sciences, Wardha, Maharashtra, India who assisted us with patient recruitment and data collection

\section{REFERENCES:}

1. García-Álvarez L, Holden MT, Lindsay H, Webb CR, Brown DF, Curran MD, Walpole E, Brooks K, Pickard DJ, Teale C, Parkhill J. Meticillin-resistant Staphylococcus aureus with a novel mecA homologue in human and bovine populations in the UK and Denmark: a descriptive study. The Lancet infectious diseases. 2011 Aug 1;11(8):595-603..

2. Naves KS, Trindade NV, Gontijo Filho PP. Methicillin-resistant Staphylococcus aureus bloodstream infection: risk factors and clinical outcome in non-intensive-care units. Revista da Sociedade Brasileira de Medicina Tropical. 2012 Apr;45(2):189-93.

3. Grema HA, Geidam YA, Gadzama GB, Ameh JA, Suleiman A. Methicillin resistant Staphyloccus aureus (MRSA): a review. Adv Anim Vet Sci. 2015;3(2):79-98.

4. Köck R, Becker K, Cookson B, van GemertPijnen JE, Harbarth S, Kluytmans JA, Mielke M, Peters G, Skov RL, Struelens MJ, Tacconelli E. Methicillin-resistant Staphylococcus aureus (MRSA): burden of disease and control challenges in Europe. Eurosurveillance. $\quad 2010 \quad$ Oct 14;15(41):19688..
5. Cooper BS, Medley GF, Stone SP, Kibbler CC, Cookson BD, Roberts JA, Duckworth G, Lai R, Ebrahim S. Methicillin-resistant Staphylococcus aureus in hospitals and the community: stealth dynamics and control catastrophes. Proceedings of the National Academy of Sciences. 2004 Jul 6;101(27):10223-8..

6. Kong EF, Johnson JK, Jabra-Rizk MA. Community-associated methicillin-resistant Staphylococcus aureus: an enemy amidst us. PLoS pathogens. 2016 Oct 6;12(10):e1005837.

7. Johnson AP. Methicillin-resistant Staphylococcus aureus: the European landscape. Journal of antimicrobial chemotherapy. 2011 May 1;66(suppl_4):iv43-8.

8. Verma S, Joshi S, Chitnis V, Hemwani N, Chitnis D. Growing problem of methicillin resistant staphylococci--Indian scenario. Indian journal of medical sciences. 2000 Dec;54(12):535-40..

9. Brasel KJ, Weigelt JA. CommunityAssociated MRSA as 4. MRSA. 2016 Apr 19:46..

10. James L, Gorwitz RJ, Jones RC, Watson JT, Hageman JC, Jernigan DB, Lord Y, Caballes $\mathrm{N}$, Cortes C, Golash RG, Price JS. Methicillin-resistant Staphylococcus aureus infections among healthy full-term newborns. Archives of Disease in ChildhoodFetal and Neonatal Edition. 2008 Jan 1;93(1):F40-4..

11. Levine NS, Lindberg RB, MASON JR AD, PRUITT JR BA. The quantitative swab culture and smear: a quick, simple method for determining the number of viable aerobic bacteria on open wounds. Journal of Trauma and Acute Care Surgery. 1976 Feb 1;16(2):89-94.

12. Wayne PA. Clinical and laboratory standards institute. Performance standards for antimicrobial susceptibility testing..

13. Stapleton PD, Taylor PW. Methicillin resistance in Staphylococcus aureus: mechanisms and modulation. Science progress. $2002 \mathrm{Feb}$;85(1):57-72.

14. Velasco D, del Mar Tomas M, Cartelle M, Beceiro A, Perez A, Molina F, Moure R, Villanueva R, Bou G. Evaluation of different 
methods for detecting methicillin (oxacillin) resistance in Staphylococcus aureus. Journal of Antimicrobial Chemotherapy. 2005 Mar 1;55(3):379-82.

15. Garoy EY, Gebreab YB, Achila OO, Tekeste DG, Kesete R, Ghirmay R, Kiflay R, Tesfu T. Methicillin-resistant Staphylococcus aureus (MRSA): prevalence and antimicrobial sensitivity pattern among patients - a multicenter study in Asmara, Eritrea. Canadian Journal of Infectious Diseases and Medical Microbiology. 2019 Jan 1;2019..

16. Onwubiko NE, Sadiq NM. Antibiotic sensitivity pattern of Staphylococcus aureus from clinical isolates in a tertiary health institution in Kano, Northwestern Nigeria. Pan African Medical Journal. 2011;8(1).

17. D'Souza N, Rodrigues C, Mehta A Molecular characterization of methicillinresistant Staphylococcus aureus with emergence of epidemic clones of sequence type (ST) 22 and ST 772 in Mumbai, India. Journal of clinical microbiology. 2010 May 1;48(5):1806-11.

18. Arora S, Devi P, Arora U, Devi B. Prevalence of methicillin-resistant Staphylococcus aureus (MRSA) in a tertiary care hospital in Northern India. Journal of laboratory physicians. $2010 \mathrm{Jul} ; 2(2): 78$. 